\title{
REAL ANALYSIS RELATED TO THE MONGE-AMPÈRE EQUATION
}

\author{
LUIS A. CAFFARELLI AND CRISTIAN E. GUTIÉRREZ
}

\begin{abstract}
In this paper we consider a family of convex sets in $\mathbf{R}^{n}, \mathcal{F}=$ $\{S(x, t)\}, x \in \mathbf{R}^{n}, t>0$, satisfying certain axioms of affine invariance, and a Borel measure $\mu$ satisfying a doubling condition with respect to the family $\mathcal{F}$. The axioms are modelled on the properties of the solutions of the real Monge-Ampère equation. The purpose of the paper is to show a variant of the Calderón-Zygmund decomposition in terms of the members of $\mathcal{F}$. This is achieved by showing first a Besicovitch-type covering lemma for the family $\mathcal{F}$ and then using the doubling property of the measure $\mu$. The decomposition is motivated by the study of the properties of the linearized Monge-Ampère equation. We show certain applications to maximal functions, and we prove a John and Nirenberg-type inequality for functions with bounded mean oscillation with respect to $\mathcal{F}$.
\end{abstract}

\section{INTRODUCTION}

The properties of the solutions of strictly elliptic partial differential equations in $\mathbf{R}^{n}$ are described in terms of the geometry determined by the euclidean balls and the Lebesgue measure. The connection between the geometry, the measure and the strict ellipticity is contained in the equation

$$
\operatorname{det} D^{2}\left(\frac{|x|^{2}}{2}\right)=1
$$

i.e., the Lebesgue measure can be viewed as the Monge-Ampère measure given by a paraboloid, and the balls in $\mathbf{R}^{n}$ are the sets where the paraboloid is smaller than an affine function. In addition, the Laplacian can be viewed as the linearization of the Monge-Ampère equation. In other words, euclidean balls, Lebesgue measure and Laplacian are all implicitly contained and related in the equation above. This phenomenon can be naturally generalized in the following way. Suppose we have a convex function $\phi$ in $\mathbf{R}^{n}$ and we generate the Monge-Ampère measure

$$
\operatorname{det} D^{2} \phi=\mu \text {. }
$$

We look at the geometry given by the sets defined as follows: given a point $x \in \mathbf{R}^{n}$ let $\ell(y)$ be a supporting hyperplane of $\phi$ at the point $(x, \phi(x))$, and given $t>0$ we define the set

$$
S(x, t)=S_{\phi}(x, t)=\left\{y \in \mathbf{R}^{n}: \phi(y)<\ell(y)+t\right\} .
$$

Received by the editors December 23, 1994 and, in revised form, January 24, 1995.

1991 Mathematics Subject Classification. Primary 35J60, 42B20; Secondary 35B45, 42B25.

Key words and phrases. Convex sets, real Monge-Ampère equation, covering lemmas, realvariable theory, $B M O$. 
These sets are obtained by projecting on $\mathbf{R}^{n}$ the points on the graph of $\phi$ that are below a supporting hyperplane lifted in $t$. We shall call these sets sections. They are convex sets and can in principle give a very degenerate geometry, in the sense that they can become very narrow in certain directions as $t$ becomes small. In fact, this is the case when $\phi$ contains lines in its graph. These degeneracies can be avoided by assuming the following doubling property on the measure $\mu$ : there exists a constant $C>0$ such that

$$
\mu(S(x, t)) \leq C \mu\left(\frac{1}{2} S(x, t)\right),
$$

for every section $S(x, t)$, where $\frac{1}{2} S(x, t)$ denotes the $\frac{1}{2}$-dilation of the set $S(x, t)$ with respect to its center of mass. In fact, it is proved in $[\mathbf{C a 2}]$ that if $\mu$ satisfies the doubling condition above, then the graph of $\phi$ does not contain lines, and the sets $S(x, t)$ are of a size that can be controlled by euclidean balls when these sets are re-scaled by using affine transformations. This is true in an essential way because the equation (1-1) is invariant under affine transformations. On the other hand, if one looks at the linearization of the equation (1-1) then the equation obtained is in general not strictly elliptic, but it is elliptic and in non-divergence form. More precisely, if one considers for a function $u(x)$ the $\operatorname{det} D^{2}(\phi+t u)$, it is easy to see that

$$
\operatorname{det} D^{2}(\phi+t u)=\operatorname{det} D^{2} \phi+t \operatorname{trace}\left(\Phi D^{2} u\right)+\ldots+t^{n} \operatorname{det} D^{2} u,
$$

where $\Phi=(\Phi)_{i j}$ is the matrix of the cofactors of $D^{2} \phi$. The coefficient of the linear term in this expansion is called the linearization of the equation (1-1) and it will be denoted by $L_{\phi} u$, i.e.,

$$
L_{\phi} u=\operatorname{trace}\left(\Phi D^{2} u\right)
$$

Since $D^{2} \phi$ is positive semidefinite, the matrix $\Phi=(\Phi)_{i j}$ is also positive semidefinite and consequently the operator $L_{\phi}$ is elliptic, possibly degenerate. In other words, the linear operator $L_{\phi}$ plays the role of a Laplacian associated with the equation (1-1).

Our purpose is to study the properties of the solutions of $L_{\phi} u=0$, and we shall show that they can be naturally described in terms of the sections $S(x, t)$ defined above and the measure $\mu$. In particular, a Harnack's inequality holds in terms of the sections $S(x, t)$. The first step in this study is to determine the properties of the sections defined above under the hypothesis (1-2). Some of these properties have been established in $[\mathbf{C a 2}]$. The study of the properties of the solutions of the pde above is based in a fundamental way in a variant of the Calderón-Zygmund decomposition in terms of the sections $S(x, t)$. The purpose of this paper is to describe this decomposition and show some applications to real analysis. The proof of the Harnack inequality will be the subject of a forthcoming paper.

The point of view taken in this paper is axiomatic: we assume that an abstract family of convex sets $S(x, t)$ is given and they satisfy certain properties. Basically, all we ask from this family is that when one section $S_{0}$ is renormalized to be equivalent to a ball, all those smaller sections that intersect $S_{0}$ deteriorate polynomially, i.e., the space is locally isotropic after renormalization. From these properties we shall prove a Calderón-Zygmund decomposition of open sets in $\mathbf{R}^{n}$ in which the classical cubes are replaced by the given family. This decomposition has independent interest and this is the reason we presented it separately. In the particular 
case of having sets coming from the equation (1-1), the decomposition plays a key role in the proof of Harnack's inequality for non-negative solutions of $L_{\phi} u=0$. In fact, it is to used to show that the distribution function (at altitude $t$ ) of a super solution decays like a negative power of $t$, see [Ca1], Lemma 6 for the elliptic case.

The paper is organized as follows. In $\S 2$, we define the class of sets we shall work with and postulate their properties. In $\S 3$, we prove a Besicovitch-type covering lemma; this lemma is purely geometric and with no reference to measure. In $\S 4$, we introduce a doubling measure on the sections given in $\S 2$ and prove the decomposition. In $\S 5$ and $\S 6$, we show certain applications of the decomposition to maximal functions, and we prove a John and Nirenberg-type exponential inequality for functions in a BMO space defined in terms of the sections.

\section{Properties of the SeCtions AND the main Result}

In order to motivate and postulate the properties of the class of convex sets which we are going to work with, we begin by mentioning a geometric lemma due to Fritz John: Let $S$ be a convex set in $\mathbf{R}^{n}$ with non-empty interior, and let $E$ be the ellipsoid of minimum volume containing $S$ whose center is the center of mass of $S$. There exists a constant $\alpha(n)$ depending only on dimension such that

$$
\alpha(n) E \subset S \subset E,
$$

where $\alpha(n) E$ means the $\alpha(n)$-dilation of $E$ with respect to its center.

Since $E$ is an ellipsoid, there is an affine transformation $T$ such that $T(E)=$ $B(0,1)$. In other words, this lemma says that every convex set $S$ with non-empty interior is "similar" to the unit ball at certain scale, i.e., there exists an affine transformation $T$ such that

$$
B(0, \alpha(n)) \subset T(S) \subset B(0,1)
$$

Given a convex set $S$ with non-empty interior, the set $T(S)$ shall be called a normalization of $S$, and $T$ shall be called an affine transformation that normalizes $S$.

The equation (1-1) is invariant by affine transformations of determinant 1 . In fact, if $T: \mathbf{R}^{n} \rightarrow \mathbf{R}^{n}$ is an affine transformation, i.e., $T x=A x+b$ where $A$ is an $n \times n$ real matrix and $b \in \mathbf{R}^{n}$, and for $\lambda>0$ we set

$$
\psi(y)=\phi_{\lambda}(T y)
$$

where $\phi$ satisfies (1-1), then $\psi_{\lambda}$ satisfies the equation

$$
M \psi_{\lambda}(y)=\operatorname{det}\left(D^{2} \psi_{\lambda}\right)(y)=\frac{1}{\lambda^{n}}(\operatorname{det} T)^{2} \mu(T y)=\bar{\mu}(y)
$$

If $\mu$ satisfies (1-2), then by the definition of section it can be shown that the new measure $\bar{\mu}$ is doubling with respect to the sections of $\psi$, i.e.,

$$
\bar{\mu}\left(S_{\psi}(x, t)\right) \leq C \bar{\mu}\left(\frac{1}{2} S_{\psi}(x, t)\right)
$$


with the same constant as in (1-2). Moreover, the sections of $\phi$ and $\psi_{\lambda}$ are related in the following way. If $h(x, y)$ is a supporting hyperplane to $\phi$ at the point $x$, then

$$
\tilde{h}\left(T^{-1} x, y\right)=\lambda^{-1} h(x, T y)
$$

is a supporting hyperplane to $\psi_{\lambda}$ at the point $T^{-1} x$. This implies that the sections of $\phi$ and $\psi_{\lambda}$ are related by the the following useful formula:

$$
T^{-1}\left(S_{\phi}(x, t)\right)=S_{\psi_{\lambda}}\left(T^{-1} x, \lambda t\right)
$$

We may rewrite this formula in the following way: if $T: \mathbf{R}^{n} \rightarrow \mathbf{R}^{n}$ is an affine transformation and we set

$$
\psi_{\lambda}(y)=\frac{1}{\lambda} \phi\left(T^{-1} y\right)
$$

then

$$
T\left(S_{\phi}(x, t)\right)=S_{\psi_{\lambda}}(T x, \lambda t) .
$$

In particular, this formula says that a normalization of a section corresponds to a normalization of the equation (1-1). In other words, if we normalize a section of $\phi$, we then a obtain section corresponding to another solution of an equation of the form (1-1). These facts and the properties of the sections $S(x, t)$ proved in [Ca2] lead us to consider a general family of convex sets with the following properties.

For each $x \in \mathbf{R}^{n}$ we have a one-parameter family of open and bounded convex sets denoted by $S(x, t), t>0, S(x, t) \subset S\left(x, t^{\prime}\right)$, for $t \leq t^{\prime}$, which shall be called sections, and satisfy:

(A) There exist constants $K_{1}, K_{2}, K_{3}$ and $\epsilon_{1}, \epsilon_{2}$, all positive, and with the following property:

given two sections $S\left(x_{0}, t_{0}\right), S(x, t)$ with $t \leq t_{0}$ such that

$$
S\left(x_{0}, t_{0}\right) \cap S(x, t) \neq \emptyset,
$$

and given $T$ an affine transformation that "normalizes" $S\left(x_{0}, t_{0}\right)$, i.e.,

$$
B(0,1 / n) \subset T\left(S\left(x_{0}, t_{0}\right)\right) \subset B(0,1),
$$

there exists $z \in B\left(0, K_{3}\right)$ depending on $S\left(x_{0}, t_{0}\right)$ and $S(x, t)$, such that

$$
B\left(z, K_{2}\left(\frac{t}{t_{0}}\right)^{\epsilon_{2}}\right) \subset T(S(x, t)) \subset B\left(z, K_{1}\left(\frac{t}{t_{0}}\right)^{\epsilon_{1}}\right),
$$

and

$$
T x \in B\left(z, \frac{1}{2} K_{2}\left(\frac{t}{t_{0}}\right)^{\epsilon_{2}}\right) .
$$

Here $B(x, t)$ denotes the euclidean ball centered at the point $x$ with radius $t$.

(B) There exists a constant $\delta>0$ such that given a section $S(x, t)$ and $y \notin S(x, t)$, if $T$ is an affine transformation that "normalizes" $S(x, t)$, then

$$
B\left(T(y), \epsilon^{\delta}\right) \cap T(S(x,(1-\epsilon) t))=\emptyset,
$$

for any $0<\epsilon<1$.

(C) $\bigcap_{t>0} S(x, t)=\{x\}$ and $\bigcup_{t>0} S(x, t)=\mathbf{R}^{n}$. 
Condition (A) can be phrased in the following way: if two sections intersect, then if we normalize the "larger" of the two, the other one looks like a ball with proportional radius at the scale in which the "larger" is normalized.

In addition, we assume that a Borel measure $\mu$ finite on compact sets is given, $\mu\left(\mathbf{R}^{n}\right)=+\infty$, and satisfies the following doubling property with respect to the parameter:

$$
\mu(S(x, 2 t)) \leq C \mu(S(x, t)) .
$$

In the case that the sections $S(x, t)$ are coming from the Monge-Ampère equation then condition (2-3) is implied by (1-2).

The main result of this paper is the following decomposition.

Theorem. Let $S(x, t)$ be an abstract family of sections satisfying properties (A), (B) and (C). Let $A$ be a bounded open subset of $\mathbf{R}^{n}$, and $0<\delta<1$. There exists a countable family of sections $\left\{S_{k}=S\left(x_{k}, t_{k}\right)\right\}_{k=1}^{\infty}, x_{k} \in A$, and $t_{k} \leq M$ with the following properties:

(a) $\frac{\delta}{C_{1}} \leq \frac{\mu\left(S_{k} \cap A\right)}{\mu\left(S_{k}\right)} \leq \delta, C_{1}>0$ depending only on $C$ in $(2-3)$.

(b) $A \subset \bigcup_{k} S_{k}$.

(c) $\mu(A) \leq \delta_{0} \mu\left(\bigcup_{1}^{\infty} S_{k}\right)$, where $\delta_{0}=\delta_{0}\left(\delta, C_{2}\right)<1$, and $C_{2}$ is a constant depending only on the parameters in (2-3), (A) and (B), and dimension.

(d) If $\tau>0$ is sufficently small and $S_{k}^{\tau}=S\left(x_{k},(1-\tau) t_{k}\right)$, then

$$
\sum_{k=1}^{\infty} \chi_{S\left(x_{k},(1-\tau) t_{k}\right)}(x) \leq K \log \frac{1}{\tau}
$$

where $K$ is a constant depending only on the constants in (A) and (B).

Remark. By the example (b) in $\S 8.8$, page 40 of $[\mathbf{S t}]$, there exists an absolutely continuous doubling measure (with respect to euclidean balls in $\mathbf{R}^{n}$ ) $\mu=f d x$, where $f$ vanishes on a set of positive measure. Therefore, the theorem above cannot hold for arbitrary bounded measurable sets $A$.

\section{A BeSICOVITCH-TYPE COVERING LEMMA FOR SECTIONS}

It is well-known that the Besicovitch covering lemma does not hold for general families of convex sets. However, for the convex sets $S(x, t)$ we have the following lemma.

Lemma 1. Let $A \subset \mathbf{R}^{n}$ be a bounded set. Suppose that for each $x \in A$ a section $S(x, t)$ is given such that $t$ is bounded by a fixed number $M$. Let us denote by $\mathcal{F}$ the family of all these sections. Then there exists a countable subfamily of $\mathcal{F}$, $\left\{S\left(x_{k}, t_{k}\right)\right\}_{k=1}^{\infty}$, with the following properties:

(i) $A \subset \bigcup_{k=1}^{\infty} S\left(x_{k}, t_{k}\right)$.

(ii) $x_{k} \notin \bigcup_{j<k}^{\infty} S\left(x_{j}, t_{j}\right), \quad \forall k \geq 2$.

(iii) For $\epsilon>0$ small (smallness depending only on the constants in (A) and (B)) we have that the family

$$
\mathcal{F}_{\epsilon}=\left\{S\left(x_{k},(1-\epsilon) t_{k}\right)\right\}_{k=1}^{\infty}
$$


has bounded overlaps; more precisely

$$
\sum_{k=1}^{\infty} \chi_{S\left(x_{k},(1-\epsilon) t_{k}\right)}(x) \leq K \log \frac{1}{\epsilon}
$$

where $K$ is a constant depending only the constants in (A) and (B); $\chi_{E}$ denotes the characteristic function of the set $E$.

Proof. We may assume $M=\sup \{t: S(x, t) \in \mathcal{F}\}$. Let

$$
\mathcal{F}_{0}=\left\{S(x, t): \frac{M}{2}<t \leq M, \quad S(x, t) \in \mathcal{F}\right\}
$$

and

$$
A_{0}=\left\{x: S(x, t) \in \mathcal{F}_{0}\right\} .
$$

Pick $S\left(x_{1}, t_{1}\right) \in \mathcal{F}_{0}$ such that $t_{1}>\frac{3}{4} M$. Then either $A_{0} \backslash S\left(x_{1}, t_{1}\right)=\emptyset$ or $A_{0} \backslash S\left(x_{1}, t_{1}\right) \neq \emptyset$. In the first case, $A_{0} \subset S\left(x_{1}, t_{1}\right)$ and we stop. In the second case, the set

$$
\left\{t: S(x, t) \in \mathcal{F}_{0} \text { and } x \in A_{0} \backslash S\left(x_{1}, t_{1}\right)\right\}
$$

is non-empty and let $\alpha_{2}$ denote its supremum. Pick $t_{2}$ in this set such that $\alpha_{2} \geq t_{2}>$ $\frac{3}{4} \alpha_{2}$ and let $S\left(x_{2}, t_{2}\right)$ be the corresponding section. We then have $x_{2} \notin S\left(x_{1}, t_{1}\right)$ and $t_{1}>\frac{3}{4} M \geq \frac{3}{4} \alpha_{2}>\frac{3}{4} t_{2}$. Again, we have either $A_{0} \backslash\left(S\left(x_{1}, t_{1}\right) \cup S\left(x_{2}, t_{2}\right)\right)=\emptyset$ or $A_{0} \backslash\left(S\left(x_{1}, t_{1}\right) \cup S\left(x_{2}, t_{2}\right)\right) \neq \emptyset$. In the first case, we have $A_{0} \subset S\left(x_{1}, t_{1}\right) \cup S\left(x_{2}, t_{2}\right)$ and we stop. In the second case, we continue the process. In general, for the $j$ th-stage we pick $t_{j}$ such that $\alpha_{j} \geq t_{j}>\frac{3}{4} \alpha_{j}$ where

$$
\alpha_{j}=\sup \left\{t: S(x, t) \in \mathcal{F}_{0} \text { and } x \in A_{0} \backslash \bigcup_{i<j} S\left(x_{i}, t_{i}\right)\right\}
$$

and select $S\left(x_{j}, t_{j}\right)$. We have $t_{i}>\left(\frac{3}{4}\right)^{j-i} t_{j}$ for $j>i$. Continuing in this way we construct a family, possibly infinite, which we denote by

$$
\mathcal{F}_{0}^{\prime}=\left\{S\left(x_{k}^{0}, t_{k}^{0}\right)\right\}_{k=1}^{\infty}
$$

with

$$
x_{j}^{0} \in A_{0} \backslash \bigcup_{i<j} S\left(x_{i}^{0}, t_{i}^{0}\right) .
$$

We now consider the family $\mathcal{F}_{1}=\left\{S(x, t): \frac{M}{4}<t \leq \frac{M}{2}\right\}$. Let

$$
A_{1}=\left\{x: S(x, t) \in \mathcal{F}_{1} \quad \text { and } \quad x \notin \bigcup_{i=1}^{\infty} S\left(x_{i}^{0}, t_{i}^{0}\right)\right\}
$$

We repeat the construction above for the set $A_{1}$ obtaining a family of sections denoted by

$$
\mathcal{F}_{1}^{\prime}=\left\{S\left(x_{i}^{1}, t_{i}^{1}\right)\right\}_{i=1}^{\infty} .
$$


We continue this process and in the $k$ th-stage we consider the family $\mathcal{F}_{k}=\{S(x, t)$ : $\left.\frac{M}{2^{k+1}}<t \leq \frac{M}{2^{k}}\right\}$ and the set

$$
A_{k}=\left\{x: S(x, t) \in \mathcal{F}_{k} \quad \text { and } \quad x \notin \bigcup \text { sections previously selected }\right\} .
$$

In the same way as before we obtain a family of sections denoted by

$$
\mathcal{F}_{k}^{\prime}=\left\{S\left(x_{i}^{k}, t_{i}^{k}\right)\right\}_{i=1}^{\infty} .
$$

Obviously, each section $S\left(x_{i}^{k}, t_{i}^{k}\right)$ in the generation $\mathcal{F}_{k}^{\prime}$ has the property that

$$
\frac{M}{2^{k+1}}<t_{i}^{k} \leq \frac{M}{2^{k}}
$$

We claim that the collection of all the sections in all generations $\mathcal{F}_{k}^{\prime}, k \geq 0$, is the family that satisfies the conclusions of Lemma 1.

To show (i), we shall first prove that each generation $\mathcal{F}_{k}^{\prime}$ has overlapping bounded by a constant depending only on the parameters in (A), and independent of $k$ and $M$. Second, we shall deduce from this that each generation $\mathcal{F}_{k}^{\prime}$ has a finite number of members; in particular, by relabeling the members of $\mathcal{F}_{k}^{\prime}$ we obtain (ii). This implies that the process in the construction of $\mathcal{F}_{k}^{\prime}$ stopped at some point and therefore all the points of $A_{k}$ are covered by the union of $\mathcal{F}_{k}^{\prime}$. Consequently, (i) follows.

Let us then show that each generation $\mathcal{F}_{k}^{\prime}$ has bounded overlapping. Suppose that

$$
z_{0} \in S\left(x_{j_{1}}^{k}, t_{j_{1}}^{k}\right) \cap \ldots \cap S\left(x_{j_{N}}^{k}, t_{j_{N}}^{k}\right),
$$

with $S\left(x_{j_{i}}^{k}, t_{j_{i}}^{k}\right) \in \mathcal{F}_{k}^{\prime}$. To simplify the notation we set $x_{j_{i}}^{k}=x_{i}, t_{j_{i}}^{k}=t_{i}$, and let $t_{0}$ be the maximum of all these $t_{i}, 1 \leq i \leq N$. We may assume by construction that $x_{l} \notin S\left(x_{i}, t_{i}\right)$ for $l>i$. By (A) we have that

$$
B\left(z_{i}, K_{2}\left(\frac{t_{i}}{t_{0}}\right)^{\epsilon_{2}}\right) \subset T\left(S\left(x_{i}, t_{i}\right)\right) \subset B\left(z_{i}, K_{1}\left(\frac{t_{i}}{t_{0}}\right)^{\epsilon_{1}}\right),
$$

and

$$
T\left(x_{i}\right) \in B\left(z_{i}, \frac{1}{2} K_{2}\left(\frac{t_{i}}{t_{0}}\right)^{\epsilon_{2}}\right)
$$

for $1 \leq i \leq N$, where $T$ is an affine transformation that normalizes $S\left(x_{0}, t_{0}\right)$. Since $\frac{M}{2^{k+1}}<t_{i} \leq \frac{M}{2^{k}}$, this implies that

$$
B\left(z_{i}, K_{2}\left(\frac{1}{2}\right)^{\epsilon_{2}}\right) \subset T\left(S\left(x_{i}, t_{i}\right)\right) \subset B\left(z_{i}, K_{1} 2^{\epsilon_{1}}\right)
$$

Since $x_{l} \notin S\left(x_{i}, t_{i}\right)$, we have that

$$
T\left(x_{l}\right) \notin T\left(S\left(x_{i}, t_{i}\right)\right), \quad l>i,
$$

and consequently,

$$
T\left(x_{l}\right) \notin B\left(z_{i}, K_{2}\left(\frac{t_{i}}{t_{0}}\right)^{\epsilon_{2}}\right), \quad l>i .
$$


This together with (3-1) implies that

$$
\left|T\left(x_{l}\right)-T\left(x_{i}\right)\right|>\frac{1}{2} K_{2}\left(\frac{1}{2}\right)^{\epsilon_{2}}, \quad l>i .
$$

Let $Q$ be the cube in $\mathbf{R}^{n}$ with center $T\left(z_{0}\right)$ and edgelength $4 K_{1} 2^{\epsilon_{1}}$. We have $T\left(S\left(x_{i}, t_{i}\right)\right) \subset Q, 1 \leq i \leq N$. We chop the cube $Q$ into $\alpha^{n}$ congruent subcubes $\tilde{Q}$ with edgelength $\frac{4 K_{1} 2^{\epsilon_{1}}}{\alpha}$. If $T\left(x_{i}\right), T\left(x_{i+l}\right) \in \tilde{Q}$ then

$$
\left|T\left(x_{i}\right)-T\left(x_{i+l}\right)\right|<\sqrt{n} \frac{4 K_{1} 2^{\epsilon_{1}}}{\alpha}
$$

If we select $\alpha$ large such that $\sqrt{n} \frac{4 K_{1} 2^{\epsilon_{1}}}{\alpha}<\frac{1}{2} K_{2}\left(\frac{1}{2}\right)^{\epsilon_{2}}$, then by (3-2) each subcube $\tilde{Q}$ contains at most one $T\left(x_{i}\right)$. Therefore, the overlapping in each generation $\mathcal{F}_{k}^{\prime}$ is at the most $\alpha^{n}$, where $\alpha$ can be taken the smallest integer bigger than $8 \sqrt{n} \frac{K_{1}}{K_{2}} 2^{\epsilon_{1}+\epsilon_{2}}$.

Let us now prove that the family $\mathcal{F}_{k}^{\prime}=\left\{S\left(x_{i}^{k}, t_{i}^{k}\right)\right\}_{i=1}^{\infty}$ is finite. We set again for simplicity $x_{i}^{k}=x_{i}$ and $t_{i}^{k}=t_{i}$. Since $A$ is bounded and $\frac{M}{2^{k+1}}<t_{i} \leq \frac{M}{2^{k}}$, the set $A_{k}$ is also bounded and by property (C) there exists a positive (large) constant $C$ such that the section $S\left(x_{1}, C t_{1}\right)$ covers $A_{k}$ and $C t_{1} \geq \frac{M}{2^{k}}$. In particular, $x_{i} \in S\left(x_{1}, C t_{1}\right)$ and then $S\left(x_{i}, t_{i}\right) \cap S\left(x_{1}, C t_{1}\right) \neq \emptyset$, and $t_{i} \leq C t_{1}$. So, we can apply (A) to obtain

$$
B\left(z_{i}, K_{2}\left(\frac{t_{i}}{C t_{1}}\right)^{\epsilon_{2}}\right) \subset T\left(S\left(x_{i}, t_{i}\right)\right) \subset B\left(z_{i}, K_{1}\left(\frac{t_{i}}{C t_{1}}\right)^{\epsilon_{1}}\right),
$$

where $T$ is an affine transformation that normalizes $S\left(x_{1}, C t_{1}\right)$, and $\left|z_{i}\right| \leq K_{3}$. We also have that

$$
\frac{1}{2 C} \leq \frac{t_{i}}{C t_{1}} \leq 1
$$

and therefore

$$
B\left(z_{i}, K_{2}\left(\frac{1}{2 C}\right)^{\epsilon_{2}}\right) \subset T\left(S\left(x_{i}, t_{i}\right)\right) \subset B\left(0, K_{1}+K_{3}\right) .
$$

Since the family $\mathcal{F}_{k}^{\prime}$ has overlapping bounded by $\alpha^{n}$, the family $T\left(S\left(x_{i}, t_{i}\right)\right)$ also has overlapping bounded by $\alpha^{n}$. Hence

$$
\sum_{i} \chi_{T\left(S\left(x_{i}, t_{i}\right)\right)}(x) \leq \alpha^{n}
$$

which implies

$$
\sum_{i} \chi_{B\left(z_{i}, K_{2}\left(\frac{1}{2 C}\right)^{\epsilon_{2}}\right)}(x) \leq \alpha^{n}
$$

Integration of this inequality over the ball $B(0, K)$, where $K=K_{1}+K_{3}$, yields

$$
\sum_{i} \omega_{n}\left(K_{2}\left(\frac{1}{2 C}\right)^{\epsilon_{2}}\right)^{n}=\sum_{i} \int_{B(0, K)} \chi_{B\left(z_{i}, K_{2}\left(\frac{1}{2 C}\right)^{\epsilon_{2}}\right)}(x) d x \leq \omega_{n} \alpha K^{n}
$$


which implies that the number of terms in the first sum is finite and we are done.

We now estimate the overlapping of sections belonging to different generations. To this end, we need to "shrink" the sections selected. Let $0<\epsilon<1$ and

$$
z_{0} \in \bigcap_{i} S\left(x_{r_{i}}^{e_{i}},(1-\epsilon) t_{r_{i}}^{e_{i}}\right)
$$

where $e_{1}<e_{2}<\ldots<e_{i}<\ldots, M 2^{-\left(e_{i}+1\right)}<t_{r_{i}}^{e_{i}} \leq M 2^{-e_{i}}$, and for simplicity in the notation we set $x_{i}=x_{r_{i}}^{e_{i}}$ and $t_{i}=t_{r_{i}}^{e_{i}}$. Fix $i$ and let $j>i$, we shall measure the gap between $e_{j}$ and $e_{i}$. Let $T_{i}$ be an affine transformation that normalizes the section $S\left(x_{i}, t_{i}\right)$. Since $j>i, t_{i} \geq(1-\epsilon) t_{j}$ and then by $(\mathrm{A})$ there exists a point $z_{i j} \in B\left(0, K_{3}\right)$ such that

$$
B\left(z_{i j}, K_{2}\left(\frac{(1-\epsilon) t_{j}}{t_{i}}\right)^{\epsilon_{2}}\right) \subset T_{i}\left(S\left(x_{j},(1-\epsilon) t_{j}\right)\right) \subset B\left(z_{i j}, K_{1}\left(\frac{(1-\epsilon) t_{j}}{t_{i}}\right)^{\epsilon_{1}}\right) .
$$

By construction $x_{j} \notin S\left(x_{i}, t_{i}\right)$. Then by (B) we have that

$$
B\left(T_{i}\left(x_{j}\right), \epsilon^{\delta}\right) \cap T_{i}\left(S\left(x_{i},(1-\epsilon) t_{i}\right)\right)=\emptyset .
$$

Consequently,

$$
\begin{aligned}
\epsilon^{\delta} & <\left|T_{i}\left(x_{j}\right)-T_{i}\left(z_{0}\right)\right| \\
& \leq\left|T_{i}\left(x_{j}\right)-z_{i j}\right|+\left|z_{i j}-T_{i}\left(z_{0}\right)\right| \\
& \leq K_{1}\left(\frac{(1-\epsilon) t_{j}}{t_{i}}\right)^{\epsilon_{1}}+K_{1}\left(\frac{(1-\epsilon) t_{j}}{t_{i}}\right)^{\epsilon_{1}} \\
& \leq 2 K_{1}\left(\frac{t_{j}}{t_{i}}\right)^{\epsilon_{1}} \\
& \leq 2^{1+\epsilon_{1}} K_{1} 2^{\left(e_{i}-e_{j}\right) \epsilon_{1}} .
\end{aligned}
$$

This implies that the number of terms in the intersection (3-3) is finite and

$$
2^{e_{j}-e_{i}} \leq 2^{1+\epsilon_{1}} K_{1} \epsilon^{-\epsilon_{1} / \delta},
$$

which clearly implies that

$$
e_{j}-e_{i} \leq C_{1} \ln \frac{1}{\epsilon}
$$

where $C_{1}$ is a constant depending only on $\epsilon_{1}, \delta$ and $K_{1}$, for all $\epsilon>0$ small, smallness depending only on the previous constants. In particular, the number of members in (3-3) is at the most $C_{1} \ln \frac{1}{\epsilon}$. Since we have proved before that the overlapping of members in the same generation is at most $\alpha^{n}$, property (iii) follows. The proof of Lemma 1 is now complete.

\section{The DECOMPOSITION}

Let $A$ be an open bounded set in $\mathbf{R}^{n}$ and for $x \in A$ consider the averages

$$
a(\lambda)=\frac{\mu(S(x, \lambda) \cap A)}{\mu(S(x, \lambda))}
$$


as a function of $\lambda$. The function $a(\lambda)$ is continuous and satisfies $a(\lambda) \rightarrow 0$ as $\lambda \rightarrow \infty$, and $a(\lambda) \rightarrow 1$ as $\lambda \rightarrow 0$.

Let $0<\delta<1$ and pick the largest $\lambda=\lambda_{x}$ with the property that $a(\lambda) \geq \delta$ and let $S(x, \lambda)$ denote the corresponding section. Let

$$
\mathcal{F}=\{S(x, \lambda)\}_{x \in A}
$$

We then have

$$
\frac{\mu(S(x, \lambda) \cap A)}{\mu(S(x, \lambda))}=\delta
$$

and

$$
\frac{\mu\left(S\left(x, \lambda^{\prime}\right) \cap A\right)}{\mu\left(S\left(x, \lambda^{\prime}\right)\right)} \leq \delta
$$

for all $\lambda^{\prime}>\lambda$. Since $\mu\left(\mathbf{R}^{n}\right)=+\infty$, by property (C) and (2-3) we have that $\lambda_{x}$ is bounded.

The family $\mathcal{F}$ is too small for our purposes; we shall enlarge it and in order to do this we prove the following lemma.

Lemma 2. Let $\mu$ be a doubling measure on the sections $S(x, \lambda)$ in the sense that there exists $C>1$ such that

$$
\mu(S(x, 2 \lambda)) \leq C \mu(S(x, \lambda)),
$$

for all $\lambda>0$, where $C$ is independent of $x$ and $\lambda$. Given $\epsilon>0$ (small) and $\lambda>0$ there exists $1<t \leq 2$, depending on $\lambda$ and $\epsilon$ such that $t-\epsilon \geq 1$ and

$$
\mu(S(x,(t-\epsilon) \lambda)) \geq(1-\epsilon \log C) \mu(S(x, t \lambda)) .
$$

Proof. Let $g(t)$ be the monotone increasing function defined by

$$
g(t)=\log (\mu(S(x, t \lambda)))
$$

and let us look at $g$ on the interval $[1,2]$. Set $g(1)=\log (\mu(S(x, \lambda)))=A$. Then by (4-2) $g(2)=\log (\mu(S(x, 2 \lambda))) \leq \log C+g(1)$. Consequently, the variation of $g$ in $[1,2]$ satisfies $V_{[1,2]} g=g(2)-g(1) \leq \log C$.

Given $\epsilon>0$ and small, divide the interval [1,2] into $N$ equal subintervals $I_{j}$ of length $\epsilon$, i.e., $N \epsilon \approx 1$. At least in one of the $I_{j}$ the variation of $g$ is $\leq \epsilon \log C$ because, if for each $j, V_{I_{j}} g>\epsilon \log C$, then

$$
N \epsilon \log C<\sum_{1}^{N} V_{I_{j}} g=V_{[1,2]} g \leq \log C,
$$

a contradiction. Therefore on some $I_{j}$, say $I_{j}=[t-\epsilon, t], 1<t \leq 2$, we have $g(t)-g(t-\epsilon) \leq \epsilon \log C$, and by exponentiating this inequality we obtain

$$
\mu(S(x,(t-\epsilon) \lambda)) \geq e^{-\epsilon \log C} \mu(S(x, t \lambda)) .
$$


The lemma now follows by observing that $e^{-s} \geq 1-s, \forall s \geq 0$.

We now enlarge the members of the family $\mathcal{F}$. Let $\epsilon>0$ (small) and for $S(x, \lambda) \in$ $\mathcal{F}$ let $1<t \leq 2$ be the number in Lemma 2. We set $r=t \lambda$, then $(t-\epsilon) \lambda=r-\epsilon \frac{r}{t} \leq$ $r-\epsilon \frac{r}{2}=r\left(1-\frac{\epsilon}{2}\right)$. This implies that

$$
S(x,(t-\epsilon) \lambda) \subset S\left(x, r\left(1-\frac{\epsilon}{2}\right)\right),
$$

and since $t-\epsilon \geq 1$ we have

$$
S(x, \lambda) \subset S\left(x, r\left(1-\frac{\epsilon}{2}\right)\right)
$$

We shall work with the families

$$
\begin{gathered}
\mathcal{G}=\{S(x, r)\}_{x \in A}, \\
\mathcal{G}_{\epsilon}=\left\{S\left(x, r\left(1-\frac{\epsilon}{2}\right)\right)\right\}_{x \in A} .
\end{gathered}
$$

Note that each member of both families satisfies (4-1), and by the doubling property $\frac{\delta}{C_{1}} \leq \frac{\mu(S \cap A)}{\mu(S)} \leq \delta$, for $S \in \mathcal{G} \cup \mathcal{G}_{\epsilon}$, where $C_{1}$ depends only on the doubling constant of $\mu$.

Proof of the Theorem. Since the set $A$ is bounded, we can apply Lemma 1 to the family $\mathcal{G}$ obtaining a countable family of sections whose members are denoted by $S_{k}=S\left(x_{k}, t_{k}\right), k=1,2, \ldots$. We also consider the family whose members are $S_{k}^{\epsilon}=S\left(x_{k},\left(1-\frac{\epsilon}{2}\right) t_{k}\right), k=1,2, \ldots$ Let $f$ be the overlapping function defined by

$$
f(x)= \begin{cases}\#\left\{k: x \in S_{k}\right\}, & \text { if } x \in \bigcup S_{k} \\ 1, & \text { if } x \notin \bigcup S_{k}\end{cases}
$$

here we have made $f \geq 1$ to prevent $f$ from vanishing. Also, denote by $f_{\epsilon}(x)$ the analogue overlapping function for the family of the $S_{k}^{\epsilon}$. Hence $1 \leq f_{\epsilon}(x) \leq f(x), \forall x$. We have the following formula:

$$
\chi_{\cup S_{k}}(x)=\frac{1}{f(x)} \sum_{1}^{\infty} \chi_{S_{k}}(x),
$$

and an analogous formula for $S_{k}^{\epsilon}$ and $f_{\epsilon}$. Since $f(x)$ may be infinite in a large region, we work with the finite family $\left\{S_{k}\right\}_{1}^{N}$ and let $N \rightarrow \infty$ at the end of the proof. Also, by Lemma 1 we have $f_{\epsilon}(x) \leq K \log (1 / \epsilon)$.

We have $A \subset \bigcup_{k} S_{k}$ and

$$
\begin{aligned}
\mu(A) & =\mu\left(\left(\bigcup S_{k}\right) \cap A\right)=\int \frac{1}{f(x)} \sum_{k} \chi_{S_{k} \cap A}(x) d \mu(x) \\
& =\sum_{k} \int \frac{1}{f(x)} \chi_{S_{k} \cap A}(x) d \mu(x) \leq \sum_{k} \int \frac{1}{f_{\epsilon}(x)} \chi_{S_{k} \cap A}(x) d \mu(x) .
\end{aligned}
$$


Also,

(4-4) $\int \frac{1}{f_{\epsilon}(x)} \chi_{S_{k}}(x) d \mu(x)=\int \frac{1}{f_{\epsilon}(x)} \chi_{S_{k} \cap A}(x) d \mu(x)+\int \frac{1}{f_{\epsilon}(x)} \chi_{S_{k} \backslash A}(x) d \mu(x)$.

Note that, $\mu\left(S_{k}\right)=\mu\left(S_{k} \backslash A\right)+\mu\left(S_{k} \cap A\right) \leq \mu\left(S_{k} \backslash A\right)+\delta \mu\left(S_{k}\right)$, i.e, $\mu\left(S_{k} \backslash A\right) \geq$ $(1-\delta) \mu\left(S_{k}\right)$. Hence,

$$
\begin{aligned}
\int \frac{1}{f_{\epsilon}(x)} \chi_{S_{k} \backslash A}(x) d \mu(x) & =\frac{1}{K \log (1 / \epsilon)} \int \frac{K \log (1 / \epsilon)}{f_{\epsilon}(x)} \chi_{S_{k} \backslash A}(x) d \mu(x) \\
\geq \frac{1}{K \log (1 / \epsilon)} \int \chi_{S_{k} \backslash A} d \mu(x) & =\frac{1}{K \log (1 / \epsilon)} \mu\left(S_{k} \backslash A\right) \\
\geq \frac{1-\delta}{K \log (1 / \epsilon)} \mu\left(S_{k}\right) & =\frac{1-\delta}{K \log (1 / \epsilon)} \int \chi_{S_{k}}(x) d \mu(x) \\
& \geq \frac{1-\delta}{K \log (1 / \epsilon)} \int \frac{1}{f_{\epsilon}(x)} \chi_{S_{k}}(x) d \mu(x) .
\end{aligned}
$$

From this inequality and (4-4) it follows that

$$
\int \frac{1}{f_{\epsilon}(x)} \chi_{S_{k} \cap A}(x) d \mu(x) \leq\left(1-\frac{1-\delta}{K \log (1 / \epsilon)}\right) \int \frac{1}{f_{\epsilon}(x)} \chi_{S_{k}}(x) d \mu(x),
$$

and consequently

$$
\mu\left(\left(\bigcup S_{k}\right) \cap A\right) \leq\left(1-\frac{1-\delta}{K \log (1 / \epsilon)}\right) \sum_{k} \int \frac{1}{f_{\epsilon}(x)} \chi_{S_{k}}(x) d \mu(x) .
$$

Now we write

$$
\begin{aligned}
& \int \frac{1}{f_{\epsilon}(x)} \chi_{S_{k}}(x) d \mu(x) \\
& =\int \frac{1}{f_{\epsilon}(x)}\left\{\chi_{S_{k}}(x)-\chi_{S_{k}^{\epsilon}}(x)\right\} d \mu(x)+\int \frac{1}{f_{\epsilon}(x)} \chi_{S_{k}^{\epsilon}}(x) d \mu(x) \\
& \leq \mu\left(S_{k}\right)-\mu\left(S_{k}^{\epsilon}\right)+\int \frac{1}{f_{\epsilon}(x)} \chi_{S_{k}^{\epsilon}}(x) d \mu(x) \\
& \leq \epsilon \log C \mu\left(S_{k}\right)+\int \frac{1}{f_{\epsilon}(x)} \chi_{S_{k}^{\epsilon}}(x) d \mu(x) \quad \text { by }(4-3) \\
& \leq \frac{\epsilon \log C}{1-\epsilon \log C} \mu\left(S_{k}^{\epsilon}\right)+\int \frac{1}{f_{\epsilon}(x)} \chi_{S_{k}^{\epsilon}}(x) d \mu(x) \\
& =\frac{\epsilon \log C}{1-\epsilon \log C} K \log (1 / \epsilon) \int \frac{1}{K \log (1 / \epsilon)} \chi_{S_{k}^{\epsilon}}(x) d \mu(x)+\int \frac{1}{f_{\epsilon}(x)} \chi_{S_{k}^{\epsilon}}(x) d \mu(x) \\
& \leq\left(1+\frac{\epsilon \log C}{1-\epsilon \log C} K \log (1 / \epsilon)\right) \int \frac{1}{f_{\epsilon}(x)} \chi_{S_{k}^{\epsilon}}(x) d \mu(x) .
\end{aligned}
$$

By adding we obtain

$$
\begin{aligned}
\sum_{k} \int \frac{1}{f_{\epsilon}(x)} \chi_{S_{k}}(x) d \mu(x) & \leq\left(1+\frac{\epsilon \log C}{1-\epsilon \log C} K \log (1 / \epsilon)\right) \int \frac{1}{f_{\epsilon}(x)} \chi_{S_{k}^{\epsilon}}(x) d \mu(x) \\
& =\left(1+\frac{\epsilon \log C}{1-\epsilon \log C} K \log (1 / \epsilon)\right) \mu\left(\bigcup S_{k}^{\epsilon}\right) .
\end{aligned}
$$


Consequently, we obtain the inequality

$$
\mu(A)=\mu\left(\left(\bigcup S_{k}\right) \cap A\right) \leq \theta(\epsilon) \mu\left(\bigcup S_{k}^{\epsilon}\right),
$$

where

$$
\theta(\epsilon)=\left(1-\frac{1-\delta}{K \log (1 / \epsilon)}\right)\left(1+\frac{\epsilon \log C}{1-\epsilon \log C} K \log (1 / \epsilon)\right) .
$$

We shall show that given $\delta, 0<\delta<1$, there exists $0<\epsilon_{0}=\epsilon_{0}(\delta, C, K)<1$ such that the function $\theta(\epsilon)$ is strictly less than 1 in the interval $\left(0, \epsilon_{0}\right]$. This together with (4-5) implies that given $0<\delta<1$ by picking $\epsilon>0$ sufficiently small there exists $\delta_{0}=\delta_{0}(\delta, K, C), 0<\delta_{0}<1$, such that

$$
\mu(A)=\mu\left(\left(\bigcup S_{k}\right) \cap A\right) \leq \delta_{0} \mu\left(\bigcup S_{k}^{\epsilon}\right),
$$

which gives the desired result.

To show the property of $\theta$, we set $\epsilon=e^{-t}, m=\log C$ and write

$$
\theta\left(e^{-t}\right)=1+\frac{m}{1-m e^{-t}} K t e^{-t}-\frac{1-\delta}{K t}-\frac{(1-\delta) m}{1-m e^{-t}} e^{-t}=1+\gamma(t) .
$$

We look for the values of $t>0$ such that $\gamma(t)<0$. These are the values for which

$$
\frac{m}{1-m e^{-t}} K t e^{-t}(K t-(1-\delta))<1-\delta .
$$

If we pick $t>0$ such that $1-m e^{-t}>1 / 2$, i.e., $t>\log (2 m)$, then the left-hand side of (4-6) is bounded by $2 m K t e^{-t}(K t-(1-\delta))$. By the fast decay of the exponential, there exists $t_{0}(\delta, m, K)$ such that

$$
2 m K t e^{-t}(K t-(1-\delta))<1-\delta,
$$

for all $t>t_{0}$. Consequently, for $t>\max \left\{\log (2 m), t_{0}(\delta, m, K)\right\}$ we have $\gamma(t)<0$. Therefore, if $0<\epsilon<\min \left\{\frac{1}{2 m}, e^{-t_{0}(\delta, m, K)}\right\}$, then $\theta(\epsilon)<1$.

\section{The maximal FunCtion}

We define the following maximal function:

$$
M f(x)=\sup _{\lambda>0} \frac{1}{\mu(S(x, \lambda))} \int_{S(x, \lambda)}|f(y)| d \mu(y),
$$

and we shall show that $M f$ is of weak-type 1-1 with respect to the measure $\mu$, i.e., there exists a constant $C>0$ such that

$$
\mu\{x: M f(x)>s\} \leq \frac{C}{s} \int_{\mathbf{R}^{n}}|f(y)| d \mu(y),
$$

for all $s>0$ and any $f \in L^{1}\left(\mathbf{R}^{n}, d \mu\right)$.

In order to apply Lemma 1 , we truncate the maximal function by setting

$$
M_{B} f(x)=\sup _{B>\lambda>0} \frac{1}{\mu(S(x, \lambda))} \int_{S(x, \lambda)}|f(y)| d \mu(y),
$$


where $B>0$, and consider the set

$$
A_{s}=\left\{|x| \leq m: M_{B} f(x)>s\right\} .
$$

Given $x \in A_{s}$ there exists $S(x, \lambda)$ such that $\lambda<B$ and

$$
\frac{1}{\mu(S(x, \lambda))} \int_{S(x, \lambda)}|f(y)| d \mu(y)>s
$$

and we call $\mathcal{F}$ the family of these sections. Given $\epsilon>0$, proceeding as in the argument after the proof of Lemma 2 we shall enlarge the family $\mathcal{F}$; i.e., given $S(x, \lambda) \in \mathcal{F}$ by Lemma 2 there exists $t=t(x, \lambda, \epsilon)$ such that $t-\epsilon \geq 1,1<t \leq 2$. We let $r=t \lambda$ and consider the family $\mathcal{F}^{\prime}=\{S(x, r)\}$. Note that

$$
S(x, \lambda) \subset S(x,(t-\epsilon) \lambda) \subset S\left(x,\left(1-\frac{\epsilon}{2}\right) r\right) \subset S(x, r) .
$$

Also, $S\left(x,\left(1-\frac{\epsilon}{2}\right) r\right) \subset S(x, 2 \lambda)$, and then by the doubling property $\mu\left(S\left(x,\left(1-\frac{\epsilon}{2}\right) r\right)\right)$ $\leq C_{1} \mu(S(x, \lambda))$. By Lemma 1 , we can select a countable subfamily of $\mathcal{F}^{\prime}$ denoted by $\left\{S\left(x_{k}, r_{k}\right)\right\}_{k=1}^{\infty}$, such that $A_{s} \subset \bigcup_{k} S\left(x_{k}, r_{k}\right)$ and by doubling we have

$$
\mu\left(A_{s}\right) \leq \sum_{k} \mu\left(S\left(x_{k}, r_{k}\right)\right) \leq C \sum_{k} \mu\left(S\left(x_{k},\left(1-\frac{\epsilon}{2}\right) r_{k}\right)\right) .
$$

Now note that by (5-1)

$$
\begin{aligned}
s & <\frac{1}{\mu(S(x, \lambda))} \int_{S(x, \lambda)}|f(y)| d \mu(y) \\
& \leq \frac{\mu\left(S\left(x,\left(1-\frac{\epsilon}{2}\right) r\right)\right)}{\mu(S(x, \lambda))} \frac{1}{\mu\left(S\left(x,\left(1-\frac{\epsilon}{2}\right) r\right)\right)} \int_{S\left(x,\left(1-\frac{\epsilon}{2}\right) r\right)}|f(y)| d \mu(y) \\
& \leq \frac{\mu(S(x, 2 \lambda))}{\mu(S(x, \lambda))} \frac{1}{\mu\left(S\left(x,\left(1-\frac{\epsilon}{2}\right) r\right)\right)} \int_{S\left(x,\left(1-\frac{\epsilon}{2}\right) r\right)}|f(y)| d \mu(y) \\
& \leq C_{1} \frac{1}{\mu\left(S\left(x,\left(1-\frac{\epsilon}{2}\right) r\right)\right)} \int_{S\left(x,\left(1-\frac{\epsilon}{2}\right) r\right)}|f(y)| d \mu(y) .
\end{aligned}
$$

This applied to $(5-2)$ yields

$$
\mu\left(A_{s}\right) \leq \frac{C}{s} \sum_{k} \int_{S\left(x_{k},\left(1-\frac{\epsilon}{2}\right) r_{k}\right)}|f(y)| d \mu(y) \leq \frac{K \log \frac{1}{\epsilon}}{s} \int_{\mathbf{R}^{n}}|f(y)| d \mu(y),
$$

by Lemma 1 .

By first letting $m \rightarrow \infty$ and then $B \rightarrow \infty$, the weak-type 1-1 of $M$ follows.

\section{The SPACE $B M O$}

Let $f$ be a real-valued function defined on $\mathbf{R}^{n}$. We say that $f \in B M O$ if there exists a constant $C>0$ such that

$$
\frac{1}{\mu(S)} \int_{S}\left|f(x)-m_{S}(f)\right| d \mu(x) \leq C
$$

for every section $S$. Here

$$
m_{S}(f)=\frac{1}{\mu(S)} \int_{S} f(x) d \mu(x) .
$$

The "norm" of $f$ in $B M O$ is the smallest constant in $C$ appearing in (6-1), and it is denoted by $\|f\|_{*}$. We shall show the following variant of the John and Nirenberg lemma. 
Lemma 3. There exist positive constants $C_{1}, C_{2}$ which depend only on the measure $\mu$ such that for every $f \in B M O$ and continuous we have

$$
\frac{1}{\mu(S)} \int_{S} \exp \left(C_{1} \frac{\left|f(x)-m_{S}(f)\right|}{\|f\|_{*}}\right) d \mu(x) \leq C_{2},
$$

for every section $S$.

Proof. We may assume that $\|f\|_{*}=1$. We shall show that there exist positive numbers $\epsilon_{0}<1$ and $M$ depending only on the measure $\mu$ such that

$$
\mu\left\{x \in S:\left|f(x)-m_{S}(f)\right|>t\right\} \leq \epsilon_{0} \mu\left\{x \in S:\left|f(x)-m_{S}(f)\right|>t-M\right\}
$$

for every section $S$ and every $t>M$. Let us fix a section $S$ and set

$$
A=\left\{x \in S:\left|f(x)-m_{S}(f)\right|>t\right\}, \quad B=\left\{x \in S:\left|f(x)-m_{S}(f)\right|>t-M\right\} .
$$

Let $0<\delta<1$ and $\left\{S\left(x_{k}, t_{k}\right)\right\}_{k=1}^{\infty}$ be the decomposition of the set $A$ given by the theorem in $\S 1$. We recall that

(1) There exists $c_{1}>0$, depending only on the doubling constant of $\mu$ such that

$$
\frac{\delta}{c_{1}}<\frac{\mu\left(S_{k} \cap A\right)}{\mu\left(S_{k}\right)} \leq \delta
$$

(2) $A \subset \bigcup_{k=1}^{\infty} S_{k}$

(3) For $0<\tau$ sufficiently small the family $S_{k}^{\tau}=S_{k}\left(x_{k},(1-\tau) t_{k}\right), k=1, \ldots$, has bounded overlaps, i.e.,

$$
\sum_{k} \chi_{S_{k}^{\tau}}(x) \leq K \log \frac{1}{\tau}
$$

and

$$
\frac{\delta}{c_{2}}<\frac{\mu\left(S_{k}^{\tau} \cap A\right)}{\mu\left(S_{k}^{\tau}\right)} \leq \delta
$$

where $c_{2}$ depends only on the doubling constant of $\mu$.

Pick $\epsilon>0$ sufficiently small such that

$$
\epsilon \leq \min \left\{\frac{\delta}{c_{1}}, \frac{\delta}{c_{2}}\right\}<\delta \leq 1-\epsilon
$$

Then we have

$$
\epsilon<\frac{\mu\left(S_{k} \cap A\right)}{\mu\left(S_{k}\right)}, \frac{\mu\left(S_{k}^{\tau} \cap A\right)}{\mu\left(S_{k}^{\tau}\right)}<1-\epsilon
$$

We claim that

$$
\frac{\mu\left(S_{k}^{\tau} \cap B\right)}{\mu\left(S_{k}^{\tau}\right)}>1-\frac{\epsilon}{2} \quad \forall k \geq 1 .
$$

Let us denote $g(x)=f(x)-m_{S}(f)$ and note that (6-3) is equivalent to

$$
\frac{\mu\left(S_{k}^{\tau} \cap\{x \in S:|g(x)| \leq t-M\}\right)}{\mu\left(S_{k}^{\tau}\right)} \leq \frac{\epsilon}{2}
$$


Suppose by contradiction that the claim is false. Then there exists $m$ such that

$$
\frac{\mu\left(S_{m}^{\tau} \cap\{x \in S:|g(x)| \leq t-M\}\right)}{\mu\left(S_{m}^{\tau}\right)}>\frac{\epsilon}{2} .
$$

Note that for any section $S^{\prime}$ we have $g(x)-m_{S^{\prime}}(g)=f(x)-m_{S^{\prime}}(f)$ and consequently, $\|g\|_{*} \leq\|f\|_{*}=1$.

Let

$$
\bar{g}_{m}=\frac{1}{\mu\left(S_{m}^{\tau}\right)} \int_{S_{m}^{\tau}} g(x) d \mu(x) .
$$

We have the following possible cases:

(a) $t-\frac{M}{2} \leq\left|\bar{g}_{m}\right|<t$.

(b) $t-M<\left|\bar{g}_{m}\right|<t-\frac{M}{2}$.

(c) $\left|\bar{g}_{m}\right|>t$.

(d) $\left|\bar{g}_{m}\right|<t-M$.

In the first case we have

$$
\begin{aligned}
1 & \geq \frac{1}{\mu\left(S_{m}^{\tau}\right)} \int_{S_{m}^{\tau}}\left|g(x)-\bar{g}_{m}\right| d \mu(x) \\
& \geq \frac{1}{\mu\left(S_{m}^{\tau}\right)} \int_{S_{m}^{\tau} \cap\{x \in S:|g(x)| \leq t-M\}}|| g(x)|-| \bar{g}_{m}|| d \mu(x) \\
& \geq \frac{M}{2} \frac{\mu\left(S_{m}^{\tau} \cap\{x \in S:|g(x)| \leq t-M\}\right)}{\mu\left(S_{m}^{\tau}\right)} \\
& \geq \frac{M}{2} \frac{\epsilon}{2}
\end{aligned}
$$

by (6-5). In the second case we have

$$
\begin{aligned}
1 \geq \frac{1}{\mu\left(S_{m}^{\tau}\right)} \int_{S_{m}^{\tau}}\left|g(x)-\bar{g}_{m}\right| d \mu(x) & \geq \frac{1}{\mu\left(S_{m}^{\tau}\right)} \int_{S_{m}^{\tau} \cap A}|| g(x)|-| \bar{g}_{m}|| d \mu(x) \\
& \geq \frac{M}{2} \frac{\mu\left(S_{m}^{\tau} \cap A\right)}{\mu\left(S_{m}^{\tau}\right)} \\
& \geq \frac{M}{2} \epsilon
\end{aligned}
$$

by property (3) of the decomposition. In case (c) we have

$$
\begin{aligned}
1 & \geq \frac{1}{\mu\left(S_{m}^{\tau}\right)} \int_{S_{m}^{\tau}}\left|g(x)-\bar{g}_{m}\right| d \mu(x) \\
& \geq \frac{1}{\mu\left(S_{m}^{\tau}\right)} \int_{S_{m}^{\tau} \cap\{x \in S:|g(x)| \leq t-M\}}|| g(x)|-| \bar{g}_{m}|| d \mu(x) \\
& \geq M \frac{\mu\left(S_{m}^{\tau} \cap\{x \in S:|g(x)| \leq t-M\}\right)}{\mu\left(S_{m}^{\tau}\right)} \\
& \geq M \frac{\epsilon}{2}
\end{aligned}
$$


by (6-5). Finally, in case (d) we have

$$
\begin{aligned}
1 \geq \frac{1}{\mu\left(S_{m}^{\tau}\right)} \int_{S_{m}^{\tau}}\left|g(x)-\bar{g}_{m}\right| d \mu(x) & \geq \frac{1}{\mu\left(S_{m}^{\tau}\right)} \int_{S_{m}^{\tau} \cap A}|| g(x)|-| \bar{g}_{m}|| d \mu(x) \\
& \geq M \frac{\mu\left(S_{m}^{\tau} \cap A\right)}{\mu\left(S_{m}^{\tau}\right)} \\
& \geq M \epsilon,
\end{aligned}
$$

again by property (3) of the decomposition. Therefore, if $M$ is sufficiently large (depending only on the size of $\epsilon$ ) we get a contradiction which shows that the inequality (6-5) is impossible. This proves the claim.

We now write

$$
\mu\left(S_{k}^{\tau} \cap B\right)=\mu\left(S_{k}^{\tau} \cap\{x \in S: t-M<|g(x)| \leq t\}\right)+\mu\left(S_{k}^{\tau} \cap A\right) .
$$

Hence, by (6-3) and the choice of $\epsilon$ we obtain

$$
\left(1-\frac{\epsilon}{2}\right) \mu\left(S_{k}^{\tau}\right)<\mu\left(S_{k}^{\tau} \cap\{x \in S: t-M<|g(x)| \leq t\}\right)+(1-\epsilon) \mu\left(S_{k}^{\tau}\right),
$$

i.e.,

$$
\mu\left(S_{k}^{\tau} \cap\{x \in S: t-M<|g(x)| \leq t\}\right)>\frac{\epsilon}{2} \mu\left(S_{k}^{\tau}\right) .
$$

By adding the last inequality over $k$, using Theorem 1 and the bounded overlaps we obtain

$$
\begin{aligned}
K \log & \frac{1}{\tau}(\mu(\{x \in S:|g(x)|>t-M\})-\mu(\{x \in S:|g(x)|>t\})) \\
& =K \log \frac{1}{\tau} \mu(\{x \in S: t-M<|g(x)| \leq t\}) \\
& \geq \sum_{k} \mu\left(S_{k}^{\tau} \cap\{x \in S: t-M<|g(x)| \leq t\}\right) \\
& >\frac{\epsilon}{2} \sum_{k} \mu\left(S_{k}^{\tau}\right) \geq \frac{\epsilon}{2} \mu\left(\bigcup_{k=1}^{\infty} S_{k}^{\tau}\right) \geq \frac{\epsilon}{2} \delta_{0}^{-1} \mu(A) .
\end{aligned}
$$

Hence,

$$
\mu(A) \leq \epsilon_{0} \mu(B)
$$

with $\epsilon_{0}=\left(1+\frac{\epsilon}{2}\left(\delta_{0} K \log \frac{1}{\tau}\right)^{-1}\right)^{-1}$.

The inequality (6-2) implies that

$$
\mu(\{x \in S:|g(x)|>M+k M\}) \leq \epsilon_{0}^{k} \mu(\{x \in S:|g(x)|>M\}) \leq \epsilon_{0}^{k} \mu(S),
$$

for $k=0,1, \ldots$.

We write

$$
\begin{aligned}
\int_{S} \exp (\alpha|g(x)|) d \mu(x)=\alpha & \int_{0}^{\infty} e^{\alpha t} \mu(\{x \in S:|g(x)|>t\}) d t \\
=\alpha & \int_{0}^{M} e^{\alpha t} \mu(\{x \in S:|g(x)|>t\}) d t \\
& \quad+\alpha \int_{M}^{\infty} e^{\alpha t} \mu(\{x \in S:|g(x)|>t\}) d t=I+I I .
\end{aligned}
$$


The integral $I$ is clearly bounded by $C \mu(S)$. To estimate $I I$ we write

$$
\begin{aligned}
I I & =e^{\alpha M} \int_{0}^{\infty} e^{\alpha t} \mu(\{x \in S:|g(x)|>M+t\}) d t \\
& =e^{\alpha M} \sum_{k=0}^{\infty} \int_{k M}^{(k+1) M} e^{\alpha t} \mu(\{x \in S:|g(x)|>M+t\}) d t \\
& \leq M e^{2 \alpha M} \sum_{k=0}^{\infty} e^{\alpha k M} \mu(\{x \in S:|g(x)|>M+k M\}) \\
& \leq M e^{2 \alpha M} \sum_{k=0}^{\infty} e^{\alpha k M} \epsilon_{0}^{k} \mu(S) .
\end{aligned}
$$

Since $\epsilon_{0}<1, \epsilon_{0}=e^{-\lambda_{0}}$ with $\lambda_{0}>0$, by taking $\alpha<\lambda_{0}$ the series converges and we obtain the lemma.

\section{REFERENCES}

[Ca1] L. A. Caffarelli, Interior a Priori Estimates for Solutions of Fully Non-linear Equations, Annals of Math. 130 (1989), 189-213. MR 90i:35046

[Ca2] _ Some Regularity Properties of Solutions of Monge-Ampère Equation, Comm. on Pure and App. Math. XLIV (1991), 965-969. MR 92h:35088

[Ca3] - Boundary Regularity of Maps with Convex Potentials, Comm. on Pure and App. Math. XLV (1992), 1141-1151. MR 93k:35054

[St] E. M. Stein, Harmonic Analysis: Real-Variable Methods, Orthogonality, and Oscillatory Integrals, Princeton Math. Series \#43, Princeton U. Press, Princeton, NJ, 1993. MR 95c: 42002

School of Mathematics, Institute for Advanced Study, Princeton, New Jersey 08540 E-mail address: caffarel@math.ias.edu

Department of Mathematics, Temple University, Philadelphia, Pennsylvania 19122

E-mail address: gutier@euclid.math.temple.edu 